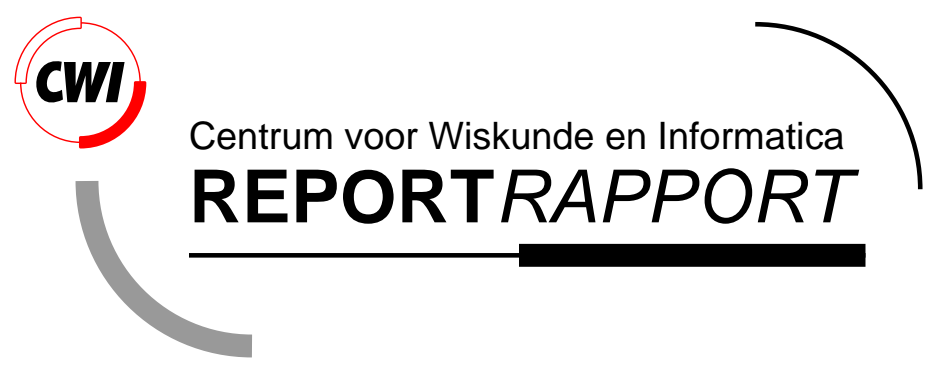

Composing morphological filters

Henk J.A.M. Heijmans

Department of Operations Reasearch, Statistics, and System Theory

BS-R9504 1995 
Report BS-R9504

ISSN 0924-0659

CWI

P.O. Box 94079

1090 GB Amsterdam

The Netherlands

$\mathrm{CWI}$ is the National Research Institute for Mathematics and Computer Science. CWI is part of the Stichting Mathematisch Centrum (SMC), the Dutch foundation for promotion of mathematics and computer science and their applications.

SMC is sponsored by the Netherlands Organization for Scientific Research (NWO). CWI is a member of ERCIM, the European Research Consortium for Informatics and Mathematics.

Copyright @ Stichting Mathematisch Centrum P.O. Box 94079, 1090 GB Amsterdam (NL) Kruislaan 413, 1098 SJ Amsterdam (NL) Telephone +3120 5929333 Telefax +3120 5924199 


\title{
Composing Morphological Filters
}

\author{
Henk J.A.M. Heijmans \\ CWI \\ P.O. Box 94079, 1090 GB Amsterdam, The Netherlands \\ henkh@cwi.nl
}

\begin{abstract}
A morphological filter is an operator on a complete lattice which is increasing and idempotent. Two well-known classes of morphological filters are openings and closings. Furthermore, an interesting class of filters, the alternating sequential filters, is obtained if one composes openings and closings. This paper explains how to construct morphological filters, and derived notions such as overfilters, underfilters, inf-overfilters, and sup-underfilters by composition, the main ingredients being dilations, erosions, openings, and closings. The class of alternating sequential filters is extended by composing overfilters and underfilters. Finally, it is shown that any composition consisting of an equal number of dilations and erosions from an adjunction is a filter. The abstract approach is illustrated with some experimental results.
\end{abstract}

AMS Subject Classification (1991): $68 \mathrm{U} 10$

Keywords \& Phrases: mathematical morphology, complete lattice, operator on a complete lattice, adjunction, opening, closing, composition of operators, morphological filter, filter derivates, alternating sequential filter, adjunctional filter.

\section{Introduction}

In mathematical morphology, a transformation or operator is called a filter if it is increasing and idempotent. Here the notion of increasingness is based on an underlying partial ordering: we will assume throughout this paper that our object space is a complete lattice. An operator $\psi$ is idempotent if $\psi^{2}=\psi$, where $\psi^{2}$ denotes the composition $\psi \psi$.

The goal of this paper is to construct morphological filters (and derived notions such as overfilters, underfilters, inf-overfilters, sup-underfilters) by means of composition. A well-known and useful class of operators obtained by composition (of openings and closings) is formed by the so-called alternating sequential filters $[2,9,10]$.

This paper starts with a short overview of the theory of morphological operators between complete lattices in Section 2. Then, in Section 3 we discuss some basic results on morphological filters and derived notions such as overfilters, underfilters, inf-overfilters, and sup-underfilters. In Section 4 we consider compositions of the type $\alpha \psi, \psi \alpha \psi, \psi \alpha, \alpha \psi \alpha$, where $\alpha$ is an opening and $\psi$ a filter or some derived notion. Subsequently, in Section 5 we consider compositions of the type $\delta^{\prime} \psi \varepsilon$ and $\varepsilon^{\prime} \psi \delta$, where $(\varepsilon, \delta)$ and $\left(\varepsilon^{\prime}, \delta^{\prime}\right)$ are adjunctions with $\delta^{\prime} \geq \delta$ and $\varepsilon^{\prime} \leq \varepsilon$. The notion of alternating sequential filter can be generalised by composing overfilters and underfilters instead of openings and closings. Details can be found in Section 6. A simple method to construct 
filters is the following: if $(\varepsilon, \delta)$ is an adjunction on the complete lattice $\mathcal{L}$ (see Section 2), then every composition of $\varepsilon$ 's and $\delta$ 's comprising an equal number of $\varepsilon$ 's and $\delta$ 's is a filter. For example, $\delta^{3} \varepsilon^{4} \delta^{2} \varepsilon^{2} \delta$ is a filter. Section 7 is devoted entirely to filters which are compositions of the operators (dilation and erosion) in an adjunction. We end with some concluding remarks, in particular on self-duality, in Section 8.

\section{Operators between complete lattices}

A complete lattice is a set $\mathcal{L}$ with a partial ordering such that every subset $\mathcal{K}$ has an infimum (greatest lower bound), denoted by $\bigwedge \mathcal{K}$, and a supremum (least upper bound), denoted by $\bigvee \mathcal{K}$. The supremum of $\mathcal{L}$ is called the greatest element and is denoted by $I$; the infimum of $\mathcal{L}$, the least element, is denoted by $O$. The lattice $\mathcal{L}$ is modular if

$$
X \vee(Y \wedge Z)=(X \vee Y) \wedge Z \text { for } X, Y, Z \in \mathcal{L} \text { with } X \leq Z
$$

Refer to [1] for a general exposition on complete lattices. Some simple illustrations of the modularity assumption can be found in $[2, \S 2.1]$.

It is generally accepted that complete lattices provide a natural framework for the study of morphological operators $[4,6,7,9,10]$. A comprehensive treatment of the complete lattice framework for morphology is given in [2]. In this section we recall some basic concepts. If $\psi$ is an operator (mapping) between $\mathcal{L}$ and $\mathcal{M}$, then we write $\psi: \mathcal{L} \rightarrow \mathcal{M}$. Let $\psi_{1}, \psi_{2}: \mathcal{L} \rightarrow \mathcal{M}$ be two operators. The (pointwise) infimum and supremum are denoted by $\psi_{1} \wedge \psi_{2}$ and $\psi_{1} \vee \psi_{2}$, respectively. If $\phi: \mathcal{L} \rightarrow \mathcal{M}$ and $\psi: \mathcal{M} \rightarrow \mathcal{N}$, then the composition $\psi \phi$ is an operator between $\mathcal{L}$ and $\mathcal{N}$ given by $(\psi \phi)(X)=\psi(\phi(X)), X \in \mathcal{L}$. For an operator $\psi$ on $\mathcal{L}$ we write $\psi^{2}=\psi \psi$.

An operator $\psi: \mathcal{L} \rightarrow \mathcal{M}$ is increasing if $X \leq Y$ (in $\mathcal{L}$ ) implies that $\psi(X) \leq \psi(Y)$ (in $\mathcal{M}$ ), for every $X, Y \in \mathcal{L}$. If $X \leq Y$ implies that $\psi(X) \geq \psi(Y)$, then $\psi$ is said to be decreasing. An operator $\psi: \mathcal{L} \rightarrow \mathcal{L}$ is called an operator on $\mathcal{L}$. The simplest operator on $\mathcal{L}$ is the identity operator id given by id $(X)=X$, for $X \in \mathcal{L}$.

2.1. Definition. An operator $\psi$ on $\mathcal{L}$ is

(a) anti-extensive if $\psi(X) \leq X$, for $X \in \mathcal{L}$;

(a') extensive if $\psi(X) \geq X$, for $X \in \mathcal{L}$;

(b) idempotent if $\psi^{2}=\psi$;

(c) an opening if $\psi$ is increasing, anti-extensive, and idempotent;

(c') a closing if $\psi$ is increasing, extensive, and idempotent.

With every complete lattice one can associate an opposite or dual complete lattice $\mathcal{L}^{\prime}$ by reversing the partial ordering: $X \leq^{\prime} Y$ in $\mathcal{L}^{\prime}$ if $X \geq Y$ in $\mathcal{L}$. This straightforward observation forms the basis of the Duality Principle, which says that every definition or statement concerning (operators on) complete lattices has a dual counterpart. For example, the dual of an opening is a closing, and vice versa. In Definition 2.1, for example, the duality between openings and closings is made explicit by using the notation (c) and $\left(\mathrm{c}^{\prime}\right)$ respectively. A major implication of the Duality Principle is that once we have demonstrated a certain property, there is no need to demonstrate also the dual property. In this paper, the Duality Principle will be exploited at several occasions, often without explicit mentioning.

From our point of view, the main theoretical concept in mathematical morphology is that of an adjunction.

2.2. Definition. A pair of operators $(\varepsilon, \delta)$, where $\varepsilon: \mathcal{L} \rightarrow \mathcal{M}$ and $\delta: \mathcal{M} \rightarrow \mathcal{L}$, is called an adjunction between $\mathcal{L}$ and $\mathcal{M}$ if

$$
\delta(Y) \leq X \Longleftrightarrow Y \leq \varepsilon(X)
$$

for every $X \in \mathcal{L}, Y \in \mathcal{M}$. 
We summarize some basic properties of adjunctions in the next two propositions.

2.3. Proposition. Let $(\varepsilon, \delta)$ be an adjunction between $\mathcal{L}$ and $\mathcal{M}$. The following are true:

(a) $(\delta, \varepsilon)$ is an adjunction between $\mathcal{M}^{\prime}$ and $\mathcal{L}^{\prime}$;

(b) for $X \in \mathcal{L}, Y \in \mathcal{M}$ :

$$
\begin{aligned}
& \delta(Y)=\bigwedge\left\{X^{\prime} \in \mathcal{L} \mid Y \leq \varepsilon\left(X^{\prime}\right)\right\} \\
& \varepsilon(X)=\bigvee\left\{Y^{\prime} \in \mathcal{M} \mid \delta\left(Y^{\prime}\right) \leq X\right\}
\end{aligned}
$$

(c) $\delta\left(\bigvee_{i \in I} Y_{i}\right)=\bigvee_{i \in I} \delta\left(Y_{i}\right)$, for every family $\left\{Y_{i} \mid i \in I\right\} \subseteq \mathcal{M}$ (in particular, $\delta$ is increasing);

(c') $\varepsilon\left(\bigwedge_{i \in I} X_{i}\right)=\bigwedge_{i \in I} \varepsilon\left(X_{i}\right)$ for every family $\left\{X_{i} \mid i \in I\right\} \subseteq \mathcal{L}$ (in particular, $\varepsilon$ is increasing);

(d) $\varepsilon \delta \varepsilon=\varepsilon$ and $\delta \varepsilon \delta=\delta$;

(e) $\delta \varepsilon$ is an opening on $\mathcal{L}$ and $\varepsilon \delta$ is a closing on $\mathcal{M}$.

An operator $\delta$ which has the property (c) above is called a dilation. An operator $\varepsilon$ with property $\left(c^{\prime}\right)$ is called an erosion. Erosions and dilations occur in pairs, as the following proposition shows.

\subsection{Proposition.}

(a) Given a dilation $\delta: \mathcal{M} \rightarrow \mathcal{L}$, there exists a unique erosion $\varepsilon: \mathcal{L} \rightarrow \mathcal{M}$, given by $(2.2)$, such that $(\varepsilon, \delta)$ is an adjunction between $\mathcal{L}$ and $\mathcal{M}$.

$\left(\mathrm{a}^{\prime}\right)$ Given an erosion $\varepsilon: \mathcal{L} \rightarrow \mathcal{M}$, there exists a unique dilation $\delta: \mathcal{M} \rightarrow \mathcal{L}$, given by (2.1), such that $(\varepsilon, \delta)$ is an adjunction between $\mathcal{L}$ and $\mathcal{M}$.

Next, we consider openings and closings, in this paper denoted by $\alpha$ and $\beta$, respectively.

\subsection{Proposition.}

(a) If $\alpha_{i}(i \in I)$ are openings, then $\bigvee_{i \in I} \alpha_{i}$ is an opening.

$\left(\mathrm{a}^{\prime}\right)$ If $\beta_{i}(i \in I)$ are closings, then $\bigwedge_{i \in I} \beta_{i}$ is a closing.

The invariance domain of an operator $\psi$ on $\mathcal{L}$ is defined as the set of its fixed points, also called invariants or roots:

$$
\operatorname{Inv}(\psi)=\{X \in \mathcal{L} \mid \psi(X)=X\} .
$$

Openings and closings are uniquely characterised by their invariance domain.

\subsection{Proposition.}

(a) Let $\alpha_{1}, \alpha_{2}$ be openings on $\mathcal{L}$, then $\alpha_{1} \leq \alpha_{2}$ iff $\operatorname{Inv}\left(\alpha_{1}\right) \subseteq \operatorname{Inv}\left(\alpha_{2}\right)$. In particular, $\alpha_{1}=\alpha_{2}$ iff $\operatorname{Inv}\left(\alpha_{1}\right)=\operatorname{Inv}\left(\alpha_{2}\right)$.

(a') Let $\beta_{1}, \beta_{2}$ be closings on $\mathcal{L}$, then $\beta_{1} \geq \beta_{2}$ iff $\operatorname{Inv}\left(\beta_{1}\right) \subseteq \operatorname{Inv}\left(\beta_{2}\right)$. In particular, $\beta_{1}=\beta_{2}$ iff $\operatorname{Inv}\left(\beta_{1}\right)=\operatorname{Inv}\left(\beta_{2}\right)$.

Originally, mathematical morphology was developed for binary images, which can be modeled mathematically as sets. In the continuous case one has to consider subsets of $\mathbb{R}^{d}$, and in the discrete case subsets of $\mathbb{Z}^{d}$. By the abstract notation $\mathbb{E}^{d}$ we represent either of these two spaces. (If one wants, one can think of $\mathbb{E}$ as an arbitrary Abelian group, and of $\mathbb{E}^{d}$ as the $d$-fold product $\mathbb{E} \times \cdots \times \mathbb{E}$.) For a set $X \subseteq \mathbb{E}^{d}$ and an element $h \in \mathbb{E}^{d}$ we define the translate $X_{h}$ as $X_{h}=\{x+h \mid x \in X\}$. By $\mathcal{P}\left(\mathbb{E}^{d}\right)$ we denote the power set of $\mathbb{E}^{d}$; it is well-known that this defines a complete Boolean lattice [2].

An operator $\psi: \mathcal{P}\left(\mathbb{E}^{d}\right) \rightarrow \mathcal{P}\left(\mathbb{E}^{d}\right)$ is called translation invariant if $\psi\left(X_{h}\right)=[\psi(X)]_{h}$, for every $X \subseteq \mathbb{E}^{d}$ and $h \in \mathbb{E}^{d}$. We use the following notation [2]. Let $X, A \subseteq \mathbb{E}^{d}$, then $X \oplus A$ is the Minkowski addition (dilation) given by

$$
X \oplus A=\{x+a \mid x \in X, a \in A\}=\bigcup_{a \in A} X_{a} .
$$


Dually, $X \ominus A$ is the Minkowski subtraction (erosion)

$$
X \ominus A=\bigcap_{a \in A} X_{-a} .
$$

The pair $\left(\varepsilon_{A}, \delta_{A}\right)$, where $\varepsilon_{A}(X)=X \ominus A$ and $\delta_{A}(X)=X \oplus A$ is an adjunction on $\mathcal{P}\left(\mathbb{E}^{d}\right)$. By Proposition 2.3, the composition $\delta_{A} \varepsilon_{A}$ is an opening and the composition $\varepsilon_{A} \delta_{A}$ is a closing. We introduce the following notation:

$$
\begin{aligned}
& X \circ A=\delta_{A} \varepsilon_{A}(X)=(X \ominus A) \oplus A, \\
& X \bullet A=\varepsilon_{A} \delta_{A}(X)=(X \oplus A) \ominus A .
\end{aligned}
$$

For $X \circ A$ one can derive the alternative expression

$$
X \circ A=\bigcup\left\{A_{h} \mid h \in \mathbb{E}^{d} \text { and } A_{h} \subseteq X\right\} .
$$

2.7. Lemma. Let $\delta, \varepsilon, \psi$ be increasing, translation invariant operators on $\mathcal{P}\left(\mathbb{E}^{d}\right)$, and assume that $(\varepsilon, \delta)$ is an adjunction. Then

$$
\begin{aligned}
& \psi \varepsilon \leq \varepsilon \psi \\
& \psi \delta \geq \delta \psi \\
& \delta \psi \varepsilon \leq \psi \\
& \varepsilon \psi \delta \geq \psi
\end{aligned}
$$

Proof. Every translation invariant erosion $\varepsilon$ on $\mathcal{P}\left(\mathbb{E}^{d}\right)$ is of the form

$$
\varepsilon(X)=X \ominus A=\bigcap_{a \in A} X_{-a},
$$

where $A \subseteq \mathbb{E}^{d}[2]$. Using that $\psi$ is increasing and translation invariant, we get

$$
\begin{aligned}
(\psi \varepsilon)(X) & =\psi\left(\bigcap_{a \in A} X_{-a}\right) \subseteq \bigcap_{a \in A} \psi\left(X_{-a}\right) \\
& =\bigcap_{a \in A}[\psi(X)]_{-a}=\psi(X) \ominus A \\
& =(\varepsilon \psi)(X) .
\end{aligned}
$$

Now (2.5) follows from the Duality Principle. Furthermore, (2.6) (resp. (2.7)) follows from (2.4) (resp. $(2.5))$ and the fact that $(\varepsilon, \delta)$ is an adjunction.

\section{Filter-derivates}

An increasing operator $\psi: \mathcal{L} \rightarrow \mathcal{L}$ is called a (morphological) filter if $\psi$ is increasing and idempotent. We emphasize here that this terminology is not consistent with most of the literature on signal and image processing, where the word 'filter' is used as a synonym for 'operator'. However, it is common practice in morphology to preserve the word 'filter' for the more restricted class indicated above.

If $\psi_{1}, \psi_{2}$ are filters, then $\psi:=\psi_{1} \vee \psi_{2}$ is not a filter, in general: it is increasing but not idempotent. However, half of the idempotence property is preserved: $\psi^{2} \geq \psi$. An increasing operator $\psi$ with this property is called an overfilter (see definition below). In fact, the concept of a filter forms the basis for a number of related concepts: we refer to them as filter-derivates. 
3.1. Definition. An increasing operator on $\mathcal{L}$ is called

(a) an overfilter if $\psi^{2} \geq \psi$;

$\left(\mathrm{a}^{\prime}\right)$ an underfilter if $\psi^{2} \leq \psi$;

(b) an inf-overfilter if $\psi($ id $\wedge \psi)=\psi$;

$\left(\mathrm{b}^{\prime}\right)$ a sup-underfilter if $\psi(\mathrm{id} \vee \psi)=\psi$;

(c) an inf-filter if $\psi$ is a filter and an inf-overfilter;

$\left(c^{\prime}\right)$ a sup-filter if $\psi$ is a filter and a sup-underfilter;

(d) a strong filter if $\psi$ is both a sup-filter and an inf-filter.

As could be expected from the Duality Principle, these definitions occur in pairs (but the concept of a strong filter is self-dual). It is easy to see that every increasing, extensive operator is an inf-overfilter, and that every inf-overfilter is an overfilter. To prove that an increasing operator is an inf-overfilter, one needs only demonstrate that $\psi($ id $\wedge \psi) \geq \psi$. The reverse inequality is trivial.

The set of increasing operators on $\mathcal{L}$ is denoted by $\mathcal{O}^{+}(\mathcal{L})$. A subcollection $\mathcal{Q}$ of $\mathcal{O}^{+}(\mathcal{L})$ is said to be closed under suprema if $\psi_{i} \in \mathcal{Q}, i \in I$, implies that $\bigvee_{i \in I} \psi_{i} \in \mathcal{Q}$. It is closed under composition if $\phi, \psi \in \mathcal{Q}$ implies that $\psi \phi \in \mathcal{Q}$. And finally, $\mathcal{Q}$ is said to be closed under self-composition if $\psi \in \mathcal{Q}$ implies that $\psi^{n} \in \mathcal{Q}$ for $n \geq 1$. It is evident that a collection of operators which is closed under composition is also closed under self-composition.

\subsection{Proposition.}

(a) The set of overfilters (inf-overfilters) is closed under suprema and self-composition.

$\left(\mathrm{a}^{\prime}\right)$ The set of underfilters (sup-underfilters) is closed under infima and self-composition.

The reader may refer to [9] or [2] for a proof.

The next result is well-known; see for example [9] or [2, Thm. 6.26].

\subsection{Proposition.}

(a) If $\psi$ is an inf-overfilter then id $\wedge \psi$ is an opening.

$\left(\mathrm{a}^{\prime}\right)$ If $\psi$ is a sup-underfilter then $\mathrm{id} \vee \psi$ is a closing.

Now we state a number of results, some known, some new, but all of them very easy to prove, which show how to obtain (inf-) overfilters, and (sup-) underfilters.

\subsection{Proposition.}

(a) If $\phi$ is increasing and extensive and $\psi$ is an overfilter, then $\phi \psi, \psi \phi$ are overfilters.

$\left(\mathrm{a}^{\prime}\right)$ If $\phi$ is increasing and anti-extensive and $\psi$ is an underfilter, then $\phi \psi, \psi \phi$ are underfilters.

(b) If $\phi$ is increasing and extensive and $\psi$ is an inf-overfilter, then $\phi \psi$ is an inf-overfilter.

$\left(\mathrm{b}^{\prime}\right)$ If $\phi$ is increasing and anti-extensive and $\psi$ is a sup-underfilter, then $\phi \psi$ is a sup-underfilter.

Proof. (a): If $\phi$ is extensive and $\psi$ is an overfilter, then

$$
\phi \psi \phi \psi \geq \phi \psi^{2} \geq \phi \psi
$$

hence $\phi \psi$ is an overfilter. Similarly, one proves that $\psi \phi$ is an overfilter.

(b): Assume that $\phi$ is extensive and that $\psi$ is an inf-overfilter, then

$$
\phi \psi(\mathrm{id} \wedge \phi \psi) \geq \phi \psi(\mathrm{id} \wedge \psi)=\phi \psi
$$

hence $\phi \psi$ is an inf-overfilter.

It is easy to extend the results in this proposition. For example, under the assumptions given in (b), one can show that $\psi \phi \psi$ is an inf-overfilter, too. 
3.5. Proposition. (See [2, Lemma 12.22]).

Assume that $\mathcal{L}$ is modular.

(a) If $\psi$ is an inf-overfilter then $\psi(\mathrm{id} \vee \psi)$ is an inf-overfilter.

$\left(\mathrm{a}^{\prime}\right)$ If $\psi$ is a sup-underfilter then $\psi(\mathrm{id} \wedge \psi)$ is a sup-underfilter.

\subsection{Proposition.}

(a) If $\psi$ is an inf-overfilter and $\phi \geq \mathrm{id} \wedge \psi$ then $\phi \psi$ is an inf-overfilter.

(b) If $\psi$ is an overfilter and $\phi \geq \psi$, then $\phi \psi$ and $\psi \phi$ are overfilters.

Proof. (a): See [2, Prop. 6.28(a)]. (b): If $\psi$ is an overfilter and $\phi \geq \psi$ then $\phi \psi \phi \psi \geq \phi \psi^{3} \geq \phi \psi$, hence $\phi \psi$ is an overfilter. Analogously, one shows that $\psi \phi$ is an overfilter.

3.7. Proposition. Let $(\varepsilon, \delta)$ be an adjunction on $\mathcal{L}$.

(a) If $\psi \geq \delta$ then $\psi \varepsilon$ is an inf-overfilter.

(a') If $\psi \leq \varepsilon$ then $\psi \delta$ is a sup-underfilter.

(b) If $\psi \geq \varepsilon$ then $\delta \psi$ is an overfilter.

$\left(\mathrm{b}^{\prime}\right)$ If $\psi \leq \delta$ then $\varepsilon \psi$ is an underfilter.

Proof. (a): See [2, Prop. 6.28(b)]. (b): If $\psi \geq \varepsilon$, then $\delta \psi \delta \psi \geq \delta \varepsilon \delta \psi=\delta \psi$, hence $\delta \psi$ is an overfilter.

Note that under the assumptions stated in (b), $\varepsilon \psi \geq \mathrm{id}$, and hence an overfilter.

For completeness we mention the following result which was first given in $[9, \S 8.4]$; see also [2, Remark 13.39(b)].

3.8. Proposition. Assume that $\mathcal{L}$ is modular. If $\phi$ is a sup-underfilter, $\psi$ an inf-overfilter, and $\phi \leq \psi$, then $\gamma=(\mathrm{id} \wedge \psi) \vee \phi$ is a filter.

We point out that $\gamma=(\mathrm{id} \wedge \psi) \vee \phi$, where $\phi \leq \psi$, is called the centre operator of $\phi$ and $\psi[2,9]$.

\section{Compositions with openings and closings}

Probably the most well-known method to construct morphological filters other than openings and closings, is by means of compositions of members of these two classes. We recall the following results $[2,9,10]$.

4.1. Proposition. Assume that $\alpha$ is an opening and that $\beta$ is a closing. The compositions

$$
\alpha \leq \alpha \beta \alpha \leq\left\{\begin{array}{l}
\beta \alpha \\
\alpha \beta
\end{array}\right\} \leq \beta \alpha \beta \leq \beta
$$

are filters. Moreover, $\alpha \beta$ and $\beta \alpha \beta$ are sup-filters and $\beta \alpha$ and $\alpha \beta \alpha$ are inf-filters.

In Section 6 we show that this result can be extended by using overfilters and underfilters. It has been shown $[2,9]$ that every inf-filter is of the form $\beta \alpha$, and, dually, that every sup-filter is of the form $\alpha \beta$. We still get interesting results if $\beta$ is replaced by an arbitrary (increasing) extensive operator.

4.2. Proposition. Assume that $\psi$ is extensive. Then $\alpha \psi, \psi \alpha \psi$ are overfilters and $\psi \alpha, \alpha \psi \alpha$ are inf-overfilters.

Proof. We show that $\psi \alpha$ is an inf-overfilter.

$$
\psi \alpha(\mathrm{id} \wedge \psi \alpha) \geq \psi \alpha(\mathrm{id} \wedge \alpha)=\psi \alpha \alpha=\psi \alpha .
$$

The proofs of the other statements are similar and as such they are left to the reader.

Next we consider the case where $\psi$ is an underfilter and sup-underfilter, respectively. 


\subsection{Proposition.}

(a) If $\psi$ is an underfilter, then $\alpha \psi, \psi \alpha \psi, \psi \alpha, \alpha \psi \alpha$ are underfilters.

(b) If $\psi$ is a sup-underfilter then $\alpha \psi, \psi \alpha \psi$ are sup-underfilters.

Proof. (a): Straightforward.

(b): We prove that $\psi \alpha \psi$ is a sup-underfilter if $\psi$ is a sup-underfilter.

$$
\psi \alpha \psi(\text { id } \vee \psi \alpha \psi) \leq \psi \alpha \psi\left(\text { id } \vee \psi^{2}\right) \leq \psi \alpha \psi(\text { id } \vee \psi)=\psi \alpha \psi \text {. }
$$

4.4. Proposition. If $\alpha \leq \psi$ then $\alpha \psi, \psi \alpha \psi$ are overfilters and $\psi \alpha, \alpha \psi \alpha$ are inf-overfilters. If, in addition, $\psi$ is anti-extensive, then $\alpha \psi=\psi \alpha=\alpha \psi \alpha=\psi \alpha \psi=\alpha$ and $\operatorname{Inv}(\alpha) \subseteq \operatorname{Inv}(\psi)$.

Proof. Let $\alpha \leq \psi$; then $\psi \alpha$ is an inf-overfilter, for:

$$
\psi \alpha(\mathrm{id} \wedge \psi \alpha) \geq \psi \alpha\left(\mathrm{id} \wedge \alpha^{2}\right)=\psi \alpha(\mathrm{id} \wedge \alpha)=\psi \alpha^{2}=\psi \alpha
$$

If, in addition, $\psi$ is anti-extensive, then $\alpha \psi \leq \alpha$ and also $\alpha \psi \geq \alpha^{2}=\alpha$, hence $\alpha \psi=\alpha$. Similarly, one shows that $\psi \alpha, \alpha \psi \alpha$ and $\psi \alpha \psi$ are equal to $\alpha$.

Of course, one can combine these results to get new ones. For example, Proposition 4.3(a) in combination with Proposition 4.4 yields that $\alpha \psi, \psi \alpha \psi$ are filters, and that $\psi \alpha, \alpha \psi \alpha$ are inf-filters when $\psi$ is an underfilter which is $\geq \alpha$.

Given an increasing operator $\psi$ on the complete lattice $\mathcal{L}$, it is easy to find openings $\alpha$ which satisfy $\alpha \leq \psi$. Namely, if $B \in \mathcal{L}$ satisfies $\psi(B) \geq B$, then $\psi \geq \alpha_{B}$, where $\alpha_{B}$ is the structural opening given by

$$
\alpha_{B}(X)= \begin{cases}B, & \text { if } B \leq X \\ O, & \text { otherwise. }\end{cases}
$$

If $\mathcal{L}=\mathcal{P}\left(\mathbb{E}^{d}\right)$ and $\psi$ is translation invariant, then $B \subseteq \psi(B)$ implies that $B_{h} \subseteq \psi\left(B_{h}\right)$, in which case

$$
X \circ B \subseteq \psi(X), X \in \mathcal{P}\left(\mathbb{R}^{d}\right) .
$$

For, $X \circ B=\cup\left\{B_{h} \mid h \in \mathbb{E}^{d}\right.$ and $\left.B_{h} \subseteq X\right\}$ by (2.3). If $B_{h} \subseteq X$, then $B_{h} \subseteq \psi\left(B_{h}\right) \subseteq \psi(X)$.

Furthermore, we have dual statements of Propositions 4.2-4.4 for closings. For example, the dual of Proposition 4.3(b) reads as follows: "If $\psi$ is an inf-overfilter (and $\beta$ a closing), then $\beta \psi, \psi \beta \psi$ are inf-overfilters."

We summarize all the results which can be derived from the propositions above (and their duals) in Table 1. This table has to be interpreted as follows: the contents of the box in the 5th row, 2nd column means:

- if $\psi$ is a filter, then $\alpha \psi$ and $\psi \alpha \psi$ are both underfilters;

- if, in addition, $\psi \geq \alpha$, then $\alpha \psi$ and $\psi \alpha \psi$ are filters.

The contents of the box in the 5 th row, 4 th column means

- if $\psi$ is a filter, then $\beta \psi$ and $\psi \beta \psi$ are both overfilters;

- if, in addition, $\psi \leq \beta$, then $\beta \psi$ and $\psi \beta \psi$ are filters.

\section{Compositions with adjunctions}

If $(\varepsilon, \delta)$ is an adjunction between the complete lattices $\mathcal{L}$ and $\mathcal{M}$, then $\delta \varepsilon$ is an opening on $\mathcal{L}$ and $\varepsilon \delta$ a closing on $\mathcal{M}$ (Proposition 2.3(e)). Furthermore, if $\left(\varepsilon^{\prime}, \delta^{\prime}\right)$ is another adjunction such that $\varepsilon^{\prime} \leq \varepsilon$ and (hence) $\delta^{\prime} \geq \delta$, then $\delta^{\prime} \varepsilon$ is an inf-overfilter on $\mathcal{L}$, and $\varepsilon^{\prime} \delta$ is a sup-underfilter 


\begin{tabular}{|c|c|c|c|c|}
\hline$\psi$ & $\alpha \psi, \psi \alpha \psi$ & $\psi \alpha, \alpha \psi \alpha$ & $\beta \psi, \psi \beta \psi$ & $\psi \beta, \beta \psi \beta$ \\
\hline $\begin{array}{l}\text { increasing } \\
\geq \alpha \text { resp. } \leq \beta\end{array}$ & $\begin{array}{l}\text { increasing } \\
\text { overfilter }\end{array}$ & $\begin{array}{l}\text { increasing } \\
\text { inf-overfilter }\end{array}$ & $\begin{array}{l}\text { increasing } \\
\text { underfilter }\end{array}$ & $\begin{array}{l}\text { increasing } \\
\text { sup-underfilter }\end{array}$ \\
\hline $\begin{array}{l}\text { overfilter } \\
\geq \alpha \text { resp. } \leq \beta\end{array}$ & $\begin{array}{l}\text { increasing } \\
\text { overfilter }\end{array}$ & $\begin{array}{l}\text { increasing } \\
\text { inf-overfilter }\end{array}$ & $\begin{array}{l}\text { overfilter } \\
\text { filter }\end{array}$ & $\begin{array}{l}\text { overfilter } \\
\text { sup-filter }\end{array}$ \\
\hline $\begin{array}{l}\text { underfilter } \\
\geq \alpha \text { resp. } \leq \beta\end{array}$ & $\begin{array}{l}\text { underfilter } \\
\text { filter }\end{array}$ & $\begin{array}{l}\text { underfilter } \\
\text { inf-filter }\end{array}$ & $\begin{array}{l}\text { increasing } \\
\text { underfilter }\end{array}$ & $\begin{array}{l}\text { increasing } \\
\text { sup-underfilter }\end{array}$ \\
\hline $\begin{array}{l}\text { filter } \\
\geq \alpha \text { resp. } \leq \beta\end{array}$ & $\begin{array}{l}\text { underfilter } \\
\text { filter }\end{array}$ & $\begin{array}{l}\text { underfilter } \\
\text { inf-filter }\end{array}$ & $\begin{array}{l}\text { overfilter } \\
\text { filter }\end{array}$ & $\begin{array}{l}\text { overfilter } \\
\text { sup-filter }\end{array}$ \\
\hline $\begin{array}{l}\text { inf-overfilter } \\
\geq \alpha \text { resp. } \leq \beta\end{array}$ & $\begin{array}{l}\text { increasing } \\
\text { overfilter }\end{array}$ & $\begin{array}{l}\text { increasing } \\
\text { inf-overfilter }\end{array}$ & $\begin{array}{l}\text { inf-overfilter } \\
\text { inf-filter }\end{array}$ & $\begin{array}{l}\text { overfilter } \\
\text { sup-filter }\end{array}$ \\
\hline $\begin{array}{l}\text { sup-underfilter } \\
\geq \alpha \text { resp. } \leq \beta\end{array}$ & $\begin{array}{l}\text { sup-underfilter } \\
\text { sup-filter }\end{array}$ & $\begin{array}{l}\text { underfilter } \\
\text { inf-filter }\end{array}$ & $\begin{array}{l}\text { increasing } \\
\text { underfilter }\end{array}$ & $\begin{array}{l}\text { increasing } \\
\text { sup-underfilter }\end{array}$ \\
\hline $\begin{array}{l}\text { inf-filter } \\
\geq \alpha \text { resp. } \leq \beta\end{array}$ & $\begin{array}{l}\text { underfilter } \\
\text { filter }\end{array}$ & $\begin{array}{l}\text { underfilter } \\
\text { inf-filter }\end{array}$ & $\begin{array}{l}\text { inf-overfilter } \\
\text { inf-filter }\end{array}$ & $\begin{array}{l}\text { overfilter } \\
\text { sup-filter }\end{array}$ \\
\hline $\begin{array}{l}\text { sup-filter } \\
\geq \alpha \text { resp. } \leq \beta\end{array}$ & $\begin{array}{l}\text { sup-underfilter } \\
\text { sup-filter }\end{array}$ & $\begin{array}{l}\text { underfilter } \\
\text { inf-filter }\end{array}$ & $\begin{array}{l}\text { overfilter } \\
\text { filter }\end{array}$ & $\begin{array}{l}\text { overfilter } \\
\text { sup-filter }\end{array}$ \\
\hline $\begin{array}{l}\text { strong filter } \\
\geq \alpha \text { resp. } \leq \beta\end{array}$ & $\begin{array}{l}\text { sup-underfilter } \\
\text { sup-filter }\end{array}$ & $\begin{array}{l}\text { underfilter } \\
\text { inf-filter }\end{array}$ & $\begin{array}{l}\text { inf-overfilter } \\
\text { inf-filter }\end{array}$ & $\begin{array}{l}\text { overfilter } \\
\text { sup-filter }\end{array}$ \\
\hline $\begin{array}{l}\text { anti-extensive } \\
\geq \alpha \text { resp. } \leq \beta\end{array}$ & $\begin{array}{l}\text { anti-extensive } \\
=\alpha\end{array}$ & $\begin{array}{l}\text { anti-extensive } \\
=\alpha\end{array}$ & $\begin{array}{l}\text { underfilter } \\
\text { underfilter }\end{array}$ & $\begin{array}{l}\text { sup-underfilter } \\
\text { sup-underfilter }\end{array}$ \\
\hline $\begin{array}{l}\text { extensive } \\
\geq \alpha \text { resp. } \leq \beta\end{array}$ & $\begin{array}{l}\text { overfilter } \\
\text { overfilter }\end{array}$ & $\begin{array}{l}\text { inf-overfilter } \\
\text { inf-overfilter }\end{array}$ & $\begin{array}{l}\text { extensive } \\
=\beta\end{array}$ & $\begin{array}{l}\text { extensive } \\
=\beta\end{array}$ \\
\hline $\begin{array}{l}\text { opening } \\
\geq \alpha \text { resp. } \leq \beta\end{array}$ & $\begin{array}{l}\text { anti-extensive } \\
=\alpha\end{array}$ & $\begin{array}{l}\text { anti-extensive } \\
=\alpha\end{array}$ & $\begin{array}{l}\text { inf-filter } \\
\text { inf-filter }\end{array}$ & $\begin{array}{l}\text { sup-filter } \\
\text { sup-filter }\end{array}$ \\
\hline $\begin{array}{l}\text { closing } \\
\geq \alpha \text { resp. } \leq \beta\end{array}$ & $\begin{array}{l}\text { sup-filter } \\
\text { sup-filter }\end{array}$ & $\begin{array}{l}\text { inf-filter } \\
\text { inf-filter }\end{array}$ & $\begin{array}{l}\text { extensive } \\
=\beta\end{array}$ & $\begin{array}{l}\text { extensive } \\
=\beta\end{array}$ \\
\hline
\end{tabular}

Table 1. Constructing filter-derivates by compositions with openings and closings.

on $\mathcal{M}$ (Proposition 3.7). In this section we investigate the compositions $\delta \psi \varepsilon, \delta^{\prime} \psi \varepsilon, \varepsilon \psi \delta, \varepsilon^{\prime} \psi \delta$, where $\psi$ is an increasing operator. For the composition $\delta \psi \varepsilon$ and $\delta^{\prime} \psi \varepsilon$ we have to require that $\psi$ is an operator on $\mathcal{M}$, whereas for the compositions $\varepsilon \psi \delta$ and $\varepsilon^{\prime} \psi \delta$ we must require that $\psi$ is an operator on $\mathcal{L}$. Since it is always clear from the context on which space $\psi$ must act, we do not mention this explicitly in our results. Throughout this section the following assumption will be made.

5.1. Assumption. $(\varepsilon, \delta)$ and $\left(\varepsilon^{\prime}, \delta^{\prime}\right)$ are two adjunctions between $\mathcal{L}$ and $\mathcal{M}$ such that $\varepsilon^{\prime} \leq \varepsilon$ and $\delta^{\prime} \geq \delta$.

5.2. Proposition. If $\alpha$ is an opening then $\delta \alpha \varepsilon$ is an opening with invariance domain

$$
\operatorname{Inv}(\delta \alpha \varepsilon)=\delta(\operatorname{Inv}(\alpha))=\{\delta(X) \mid X \in \operatorname{Inv}(\alpha)\} .
$$

Proof. To show that the operator $\delta \alpha \varepsilon$ is an opening, we show that it is anti-extensive (hence an underfilter) and an overfilter. First, $\delta \alpha \varepsilon \leq \delta \varepsilon \leq \mathrm{id}$. Secondly, as $\varepsilon \delta \geq \mathrm{id}, \delta \alpha \varepsilon \delta \alpha \varepsilon \geq \delta \alpha^{2} \varepsilon=\delta \alpha \varepsilon$.

Concerning the invariance domain, assume that $X \in \operatorname{Inv}(\delta \alpha \varepsilon)$, hence $\delta \alpha \varepsilon(X)=X$. Since $\alpha \varepsilon(X) \in \operatorname{Inv}(\alpha)$, we have $X \in \delta(\operatorname{Inv}(\alpha))$. Conversely, if $X \in \operatorname{Inv}(\alpha)$, then $\delta \alpha \varepsilon \delta(X) \leq \delta \varepsilon \delta(X)=$ $\delta(X)$. But also, since $\varepsilon \delta \geq$ id, we get $\delta \alpha \varepsilon \delta(X) \geq \delta \alpha(X)=\delta(X)$. This yields that $\delta(X) \in$ $\operatorname{Inv}(\delta \alpha \varepsilon)$. 
Note that $\delta \alpha \varepsilon \leq \delta \varepsilon$. If, moreover, $\delta, \varepsilon, \alpha$ are translation invariant operators on $\mathcal{P}\left(\mathbb{E}^{d}\right)$ then also $\delta \alpha \varepsilon \leq \alpha$ (Lemma 2.7). In fact, in the translation invariant case a lot more can be said. For, in this case, every adjunction is of the form $\left(\varepsilon_{A}, \delta_{A}\right)$, and every opening can be written as

$$
\alpha(X)=\bigcup_{B \in \mathcal{B}} X \circ B
$$

for some collection of structuring elements $\mathcal{B}$ (see [2, Thm.4.24] or [5]). Thus, using that $\delta_{A}$ acts distributively over unions, we get

$$
\begin{aligned}
\delta_{A} \alpha \varepsilon_{A}(X) & =\left(\bigcup_{B \in \mathcal{B}}(X \ominus A) \circ B\right) \oplus A \\
& =\bigcup_{B \in \mathcal{B}}((X \ominus A) \circ B) \oplus A \\
& =\bigcup_{B \in \mathcal{B}}((X \ominus(A \oplus B)) \oplus(A \oplus B), \\
& =\bigcup_{B \in \mathcal{B}} X \circ(A \oplus B),
\end{aligned}
$$

which is a union of openings with structuring elements $A \oplus B$, where $B \in \mathcal{B}$.

\subsection{Proposition.}

(a) If $\psi$ is an overfilter then $\delta^{\prime} \psi \varepsilon$ is an overfilter.

(b) If $\psi$ is an inf-overfilter, then $\delta^{\prime} \psi \varepsilon$ is an inf-overfilter.

Proof. (a) is easy. We prove (b). As $\varepsilon$ distributes over infima we get

$$
\begin{aligned}
\delta^{\prime} \psi \varepsilon\left(\mathrm{id} \wedge \delta^{\prime} \psi \varepsilon\right) & =\delta^{\prime} \psi\left(\varepsilon \wedge \varepsilon \delta^{\prime} \psi \varepsilon\right)=\delta^{\prime} \psi\left(\text { id } \wedge \varepsilon \delta^{\prime} \psi\right) \varepsilon \\
& \geq \delta^{\prime} \psi(\text { id } \wedge \varepsilon \delta \psi) \varepsilon \geq \delta^{\prime} \psi(\text { id } \wedge \psi) \varepsilon=\delta^{\prime} \psi \varepsilon
\end{aligned}
$$

This concludes the proof.

Again, the Duality Principle yields dual results of these two propositions. For example, the dual statement of Proposition 5.3(a) reads as follows: "If $\psi$ is an underfilter, then $\varepsilon^{\prime} \psi \delta$ is an underfilter."

\begin{tabular}{|l|l|l|l|l|}
\hline$\psi$ & $\delta \psi \varepsilon$ & $\delta^{\prime} \psi \varepsilon$ & $\varepsilon \psi \delta$ & $\varepsilon^{\prime} \psi \delta$ \\
\hline overfilter & overfilter & overfilter & increasing & increasing \\
\hline underfilter & increasing & increasing & underfilter & underfilter \\
\hline filter & overfilter & overfilter & underfilter & underfilter \\
\hline inf-overfilter & inf-overfilter & inf-overfilter & increasing & increasing \\
\hline sup-underfilter & increasing & increasing & sup-underfilter & sup-underfilter \\
\hline inf-filter & inf-overfilter & inf-overfilter & underfilter & underfilter \\
\hline sup-filter & overfilter & overfilter & sup-underfilter & sup-underfilter \\
\hline strong filter & inf-overfilter & inf-overfilter & sup-underfilter & sup-underfilter \\
\hline anti-extensive & anti-extensive & increasing & sup-underfilter & sup-underfilter \\
\hline extensive & inf-overfilter & inf-overfilter & extensive & increasing \\
\hline opening & opening & inf-overfilter & sup-underfilter & sup-underfilter \\
\hline closing & inf-overfilter & inf-overfilter & closing & sup-underfilter \\
\hline$\psi=$ id & opening & inf-overfilter & closing & sup-underfilter \\
\hline
\end{tabular}

Table 2. Constructing filter-derivates by composition with adjunctions. 
Our results are summarized in Table 2. For the sake of completeness, we point out that it is not possible to derive any useful result for $\delta \psi \varepsilon^{\prime}$ and $\varepsilon \psi \delta^{\prime}$, apart from the trivial facts that $\delta \psi \varepsilon^{\prime}$ is anti-extensive if $\psi$ is anti-extensive, and that $\varepsilon \psi \delta^{\prime}$ is extensive if $\psi$ is extensive.

We conclude this section with the following result.

\subsection{Proposition.}

(a) If $\psi$ is an overfilter which is $\leq \varepsilon \delta$, for some adjunction $(\varepsilon, \delta)$, then $\delta \psi^{n} \varepsilon$ is an opening $\leq \delta \varepsilon$, for every $n \geq 1$.

$\left(\mathrm{a}^{\prime}\right)$ If $\psi$ is an underfilter which is $\geq \delta \varepsilon$, for some adjunction $(\varepsilon, \delta)$, then $\varepsilon \psi^{n} \delta$ is a closing $\geq \varepsilon \delta$, for every $n \geq 1$.

Proof. First we observe that $\psi^{n} \leq(\varepsilon \delta)^{n}=\varepsilon \delta$. To get that $\delta \psi^{n} \varepsilon$ is an opening we show that $\delta \psi^{n} \varepsilon$ is an anti-extensive overfilter. That $\delta \psi^{n} \varepsilon$ is anti-extensive (and $\leq \delta \varepsilon$ ) follows from

$$
\delta \psi^{n} \varepsilon \leq \delta \varepsilon \delta \varepsilon=\delta \varepsilon \leq \mathrm{id}
$$

Furthermore, since $\psi$ is an overfilter,

$$
\delta \psi^{n} \varepsilon \delta \psi^{n} \varepsilon \geq \delta \psi^{n} \mathrm{id} \psi^{n} \varepsilon=\delta \psi^{2 n} \varepsilon \geq \delta \psi^{n} \varepsilon,
$$

hence $\delta \psi^{n} \varepsilon$ is an overfilter. This concludes the proof.

\section{Alternating sequential filters}

In this section we discuss an important class of morphological filters, the so-called alternating sequential filters or $A S$-filters. We start with an extension of Proposition 4.1.

6.1. Proposition. Assume that $\phi$ is an overfilter, that $\psi$ is an underfilter, and that $\phi \leq \psi$. The compositions $\phi \psi, \psi \phi, \phi \psi \phi, \psi \phi \psi$ are filters, and

$$
\phi \leq \phi \psi \phi \leq\left\{\begin{array}{l}
\psi \phi \\
\phi \psi
\end{array}\right\} \leq \psi \phi \psi \leq \psi
$$

If $\phi$ is an inf-overfilter then $\psi \phi$ and $\phi \psi \phi$ are inf-filters. If $\psi$ is a sup-underfilter, then $\phi \psi$ and $\psi \phi \psi$ are sup-filters.

Proof. That, e.g., $\psi \phi$ is a filter follows from $\psi \phi \psi \phi \leq \psi^{3} \phi \leq \psi \phi$ and $\psi \phi \psi \phi \geq \psi \phi^{3} \geq \psi \phi$. Similarly one shows that the other compositions are filters, too.

Furthermore,

$$
\phi \leq \phi^{3} \leq \phi \psi \phi \leq \phi \psi^{2} \leq \phi \psi \leq \phi^{2} \psi \leq \psi \phi \psi \leq \psi^{3} \leq \psi
$$

The inequalities with $\psi \phi$ instead of $\phi \psi$ follow analogously.

Assume that $\phi$ is an inf-overfilter, then

$$
\psi \phi(\mathrm{id} \wedge \psi \phi) \geq \psi \phi\left(\mathrm{id} \wedge \phi^{2}\right) \geq \psi \phi(\mathrm{id} \wedge \phi)=\psi \phi
$$

hence the filter $\psi \phi$ is an inf-overfilter, and therefore an inf-filter. The other assertions are proved in a similar way. 
6.2. Example. Consider the translation invariant operators on $\mathcal{P}\left(\mathbb{E}^{d}\right)$. In Lemma 2.7 we have seen that

$$
\delta \psi \varepsilon \leq \psi \leq \varepsilon \psi \delta
$$

for any translation invariant operator $\psi$. Now, by Proposition 5.3, if $\psi$ is a filter, then $\delta \psi \varepsilon$ is an overfilter and $\varepsilon \psi \delta$ is an underfilter. This yields that

$$
\delta \psi \varepsilon^{2} \psi \delta^{2} \psi \varepsilon \leq\left\{\begin{array}{l}
\delta \psi \varepsilon^{2} \psi \delta \\
\varepsilon \psi \delta^{2} \psi \varepsilon
\end{array}\right\} \leq \varepsilon \psi \delta^{2} \psi \varepsilon^{2} \psi \delta
$$

are filters, too. Substituting, for example, $\psi=\delta \varepsilon$ one obtains the filters

$$
\delta^{2} \varepsilon^{3} \delta^{3} \varepsilon^{2} \leq\left\{\begin{array}{l}
\delta^{2} \varepsilon^{3} \delta \\
\varepsilon \delta^{3} \varepsilon^{2}
\end{array}\right\} \leq \varepsilon \delta^{3} \varepsilon^{3} \delta
$$

Of course, it is not difficult to find a direct proof of the latter inequalities. Moreover, the results which will be given in Section 7 yield immediately that these compositions define filters.

Consider an inf-overfilter $\phi$ of the form $\phi=\delta_{\phi}^{\prime} \varepsilon_{\phi}$, where $\left(\varepsilon_{\phi}, \delta_{\phi}\right)$ and $\left(\varepsilon_{\phi}^{\prime}, \delta_{\phi}^{\prime}\right)$ are adjunctions between $\mathcal{L}$ and some other complete lattice $\mathcal{M}_{\phi}$ such that $\delta_{\phi}^{\prime} \geq \delta_{\phi}$ and (hence) $\varepsilon_{\phi}^{\prime} \leq \varepsilon_{\phi}$. Dually, let $\psi$ be a sup-underfilter of the form $\psi=\varepsilon_{\psi}^{\prime} \delta_{\psi}$, where $\left(\varepsilon_{\psi}, \delta_{\psi}\right)$ and $\left(\varepsilon_{\psi}^{\prime}, \delta_{\psi}^{\prime}\right)$ are adjunctions between some complete lattice $\mathcal{M}_{\psi}$ and $\mathcal{L}$ such that $\delta_{\psi}^{\prime} \geq \delta_{\psi}$ and $\varepsilon_{\psi}^{\prime} \leq \varepsilon_{\psi}$. To apply Proposition 6.1 we must choose the adjunctions in such a way that

$$
\delta_{\phi}^{\prime} \varepsilon_{\phi} \leq \varepsilon_{\psi}^{\prime} \delta_{\psi}
$$

or equivalently,

$$
\delta_{\psi}^{\prime} \delta_{\phi}^{\prime} \leq \delta_{\psi} \delta_{\phi}
$$

6.3. Example. Consider the translation invariant operators on $\mathcal{P}\left(\mathbb{E}^{d}\right)$. Let $A_{\psi}$ be a structuring element in $\mathbb{E}^{d}$ such that $\delta_{\psi}(X)=X \oplus A_{\psi}$. The structuring elements $A_{\phi}, A_{\psi}^{\prime}, A_{\phi}^{\prime}$ are defined analogously. The inequality (6.1) holds if and only if

$$
A_{\psi}^{\prime} \oplus A_{\phi}^{\prime} \subseteq A_{\psi} \oplus A_{\phi}
$$

On the other hand, since $\delta_{\psi}^{\prime} \geq \delta_{\psi}$ and $\delta_{\phi}^{\prime} \geq \delta_{\phi}$, we have $A_{\psi} \subseteq A_{\psi}^{\prime}$ and $A_{\phi} \subseteq A_{\phi}^{\prime}$, which means that the reverse inclusion holds as well. Combining these facts, this means that we have to find structuring elements satisfying the relations

$$
\left\{\begin{array}{l}
A_{\phi} \subseteq A_{\phi}^{\prime} \\
A_{\psi} \subseteq A_{\psi}^{\prime} \\
A_{\phi} \oplus A_{\psi}=A_{\phi}^{\prime} \oplus A_{\psi}^{\prime}
\end{array}\right.
$$

A nontrivial solution of (6.2) is given by

$$
A_{\phi}=A_{\psi}=A=\left[\begin{array}{lll}
\bullet & \bullet & \bullet \\
\bullet & \bullet & \bullet \\
\bullet & \bullet & \bullet
\end{array}, \quad A_{\phi}^{\prime}=A_{\psi}^{\prime}=A^{\prime}=\begin{array}{lll}
\bullet & \bullet & \bullet \\
\bullet & \bullet & \bullet \\
\bullet & \bullet & \bullet
\end{array}\right.
$$

In Figure 1 we have applied the inf-filter $\psi \phi$ and the sup-filter $\phi \psi$, where $\phi(X)=(X \ominus A) \oplus A^{\prime}$ and $\psi(X)=(X \oplus A) \ominus A^{\prime}$ to a binary image $X$ (the image $X$ being represented by the black pixels). For comparison, we have depicted the inf-filter $\beta \alpha(X)$ and the sup-filter $\alpha \beta(X)$, where 


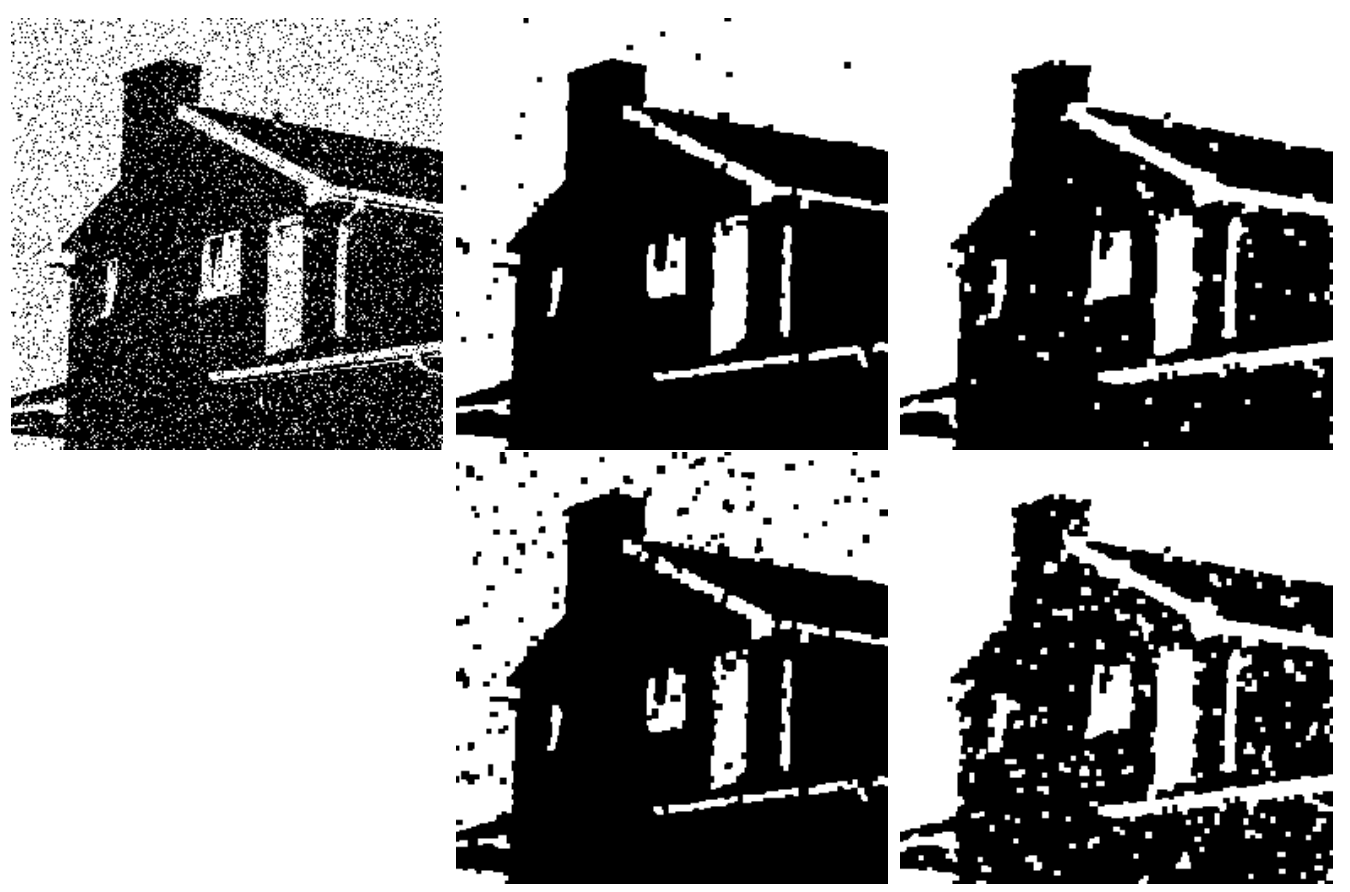

Fig. 1. First row, from left to right: the original image $X$, and the images $\phi \psi(X)$ and $\psi \phi(X)$. Second row, from left to right: the images $\alpha \beta(X)$ and $\beta \alpha(X)$.

$\alpha(X)=\left(X \ominus A^{\prime}\right) \oplus A^{\prime}$ and $\beta(X)=\left(X \oplus A^{\prime}\right) \ominus A^{\prime}$, in the second row of Figure 1. This figure illustrates clearly that the noise-cleaning effect of $\psi \phi$ and $\phi \psi$ is much greater than that of $\beta \alpha$ and $\alpha \beta$, respectively.

In the following result we generalise Proposition 6.1 .

6.4. Proposition. Let $\phi_{1}, \phi_{2}, \ldots$ be overfilters and $\psi_{1}, \psi_{2}, \ldots$ be underfilters such that

$$
\cdots \leq \phi_{2} \leq \phi_{1} \leq \psi_{1} \leq \psi_{2} \leq \cdots
$$

Every composition of operators of these two sequences which contains at least one operator from each sequence is a filter.

Proof. Consider a composition $\xi_{1} \xi_{2} \cdots \xi_{n}$ of operators of these two sequences and assume that one of the $\phi_{i}$ 's and one of the $\psi_{i}$ 's occurs in this composition. We show that this composition is an underfilter. That it is also an overfilter follows by similar arguments. Let $\xi_{j}$ be the largest of all operators in the given composition. Then $\xi_{j}$ is one of the $\psi_{k}$ 's, hence an underfilter. We have

$$
\begin{aligned}
\left(\xi_{1} \xi_{2} \cdots \xi_{n}\right)\left(\xi_{1} \xi_{2} \cdots \xi_{n}\right) & =\left(\xi_{1} \xi_{2} \cdots \xi_{j-1} \xi_{j} \xi_{j+1} \cdots \xi_{n}\right)\left(\xi_{1} \xi_{2} \cdots \xi_{j-1} \xi_{j} \xi_{j+1} \cdots \xi_{n}\right) \\
& \leq\left(\xi_{1} \xi_{2} \cdots \xi_{j-1}\right) \xi_{j}^{n+1}\left(\xi_{j+1} \cdots \xi_{n}\right) \\
& \leq \xi_{1} \xi_{2} \cdots \xi_{j-1} \xi_{j} \xi_{j+1} \cdots \xi_{n}
\end{aligned}
$$

for $\xi_{j}^{n+1} \leq \xi_{j}$ since $\xi_{j}$ is an underfilter.

6.5. Corollary. Let $\alpha_{1} \geq \alpha_{2} \geq \alpha_{3} \geq \cdots$ be openings and let $\beta_{1} \leq \beta_{2} \leq \beta_{3} \leq \cdots$ be closings. Every composition of operators of these two sequences is a filter. 
In fact, this latter result has first been established by Schonfeld and Goutsias [8].

We introduce the following notation: if $\xi_{1}, \xi_{2}, \ldots$ are operators, then

$$
(\xi)_{n}=\xi_{n} \xi_{n-1} \cdots \xi_{1}, \quad n \geq 1
$$

More generally, if $\eta_{1}, \eta_{2}, \ldots$ is another sequence of operators, then

$$
(\xi \eta)_{n}=\xi_{n} \eta_{n} \xi_{n-1} \eta_{n-1} \cdots \xi_{1} \eta_{1} \text { and }(\xi \eta \xi)_{n}=\xi_{n} \eta_{n} \xi_{n} \xi_{n-1} \eta_{n-1} \xi_{n-1} \cdots \xi_{1} \eta_{1} \xi_{1}
$$

If $\alpha_{1}, \alpha_{2}, \ldots$ is a sequence of openings which satisfy $\alpha_{1} \geq \alpha_{2} \geq \cdots$, and if $\beta_{1}, \beta_{2}, \ldots$ is a sequence of closings with $\beta_{1} \leq \beta_{2} \leq \cdots$, then the compositions $(\alpha \beta)_{n},(\beta \alpha)_{n},(\alpha \beta \alpha)_{n},(\beta \alpha \beta)_{n}$ are filters, called alternating sequential filters or AS-filters $[2,9,10]$.

As sequence of operators $\psi_{1}, \psi_{2}, \ldots$ is said to be absorbing if

$$
\psi_{n} \psi_{m}=\psi_{n}, \quad n \geq m
$$

The following result is straightforward.

6.6. Lemma. Let $\xi_{1}, \xi_{2}, \ldots$ be a family of increasing operators such that $(\xi)_{n}$ is a filter for every $n$, then the sequence $(\xi)_{n}$ is absorbing.

Proof. Let $n \geq m$, then

$$
\begin{aligned}
(\xi)_{n}(\xi)_{m} & =\xi_{n} \xi_{n-1} \cdots \xi_{m+1}(\xi)_{m}(\xi)_{m} \\
& =\xi_{n} \xi_{n-1} \cdots \xi_{m+1}(\xi)_{m} \\
& =(\xi)_{n}
\end{aligned}
$$

This proves the result.

In particular, the sequences $(\alpha \beta)_{n},(\beta \alpha)_{n},(\alpha \beta \alpha)_{n},(\beta \alpha \beta)_{n}$ are absorbing.

The next proposition shows that the classes of AS-filters obtained from families of openings and closings can be extended considerably if one uses overfilters and underfilters, respectively.

6.7. Proposition. Let $\phi_{1}, \phi_{2}, \ldots$ be overfilters and $\psi_{1}, \psi_{2}, \ldots$ be underfilters such that the following conditions are satisfied:

$$
\begin{aligned}
& \phi_{n} \leq \psi_{n}, \\
& \phi_{n} \phi_{n-1} \geq \phi_{n}, \\
& \psi_{n} \psi_{n-1} \leq \psi_{n} .
\end{aligned}
$$

Then $(\phi \psi)_{n},(\psi \phi)_{n},(\phi \psi \phi)_{n},(\psi \phi \psi)_{n}$ are absorbing sequences of filters and

$$
(\phi \psi \phi)_{n} \leq\left\{\begin{array}{c}
(\phi \psi)_{n} \\
(\psi \phi)_{n}
\end{array}\right\} \leq(\psi \phi \psi)_{n}
$$

Proof. To show that $(\phi \psi)_{n}$ is a filter we must show that it is both an underfilter and an overfilter. Note first that

$$
(\psi)_{n}=\psi_{n} \cdots \psi_{3} \psi_{2} \psi_{1} \leq \psi_{n} \cdots \psi_{3} \psi_{2} \leq \psi_{n} \cdots \psi_{3} \leq \text { etc. } \leq \psi_{n}
$$


Now

$$
\begin{aligned}
(\phi \psi)_{n}(\phi \psi)_{n} & =\phi_{n}\left(\psi_{n}(\phi \psi)_{n} \phi_{n} \psi_{n}\right)(\phi \psi)_{n-1} \\
& \leq \phi_{n}\left(\psi_{n}(\psi \psi)_{n} \psi_{n}^{2}\right)(\phi \psi)_{n-1} \\
& \leq \phi_{n}\left(\psi_{n}(\psi)_{n} \psi_{n}\right)(\phi \psi)_{n-1} \\
& \leq \phi_{n}\left(\psi_{n}^{3}\right)(\phi \psi)_{n-1} \\
& \leq \phi_{n} \psi_{n}(\phi \psi)_{n-1} \\
& =(\phi \psi)_{n}
\end{aligned}
$$

This shows that $(\phi \psi)_{n}$ is an underfilter. To show that it is also an overfilter, we note first that

$$
(\phi)_{n}=\phi_{n} \phi_{n-1} \cdots \phi_{1} \geq \phi_{n}
$$

Therefore,

$$
\begin{aligned}
(\phi \psi)_{n}(\phi \psi)_{n} & \geq(\phi \phi)_{n}(\phi \psi)_{n} \\
& \geq(\phi)_{n}(\phi \psi)_{n} \\
& \geq \phi_{n}(\phi \psi)_{n} \\
& =\phi_{n}^{2} \psi_{n}(\phi \psi)_{n-1} \\
& \geq \phi_{n} \psi_{n}(\phi \psi)_{n-1} \\
& =(\phi \psi)_{n}
\end{aligned}
$$

Thus $(\phi \psi)_{n}$ is a filter.

To show that the other three expressions are filters, one can use similar arguments.

Furthermore, e.g.,

$$
(\phi \psi \phi)_{n} \leq(\phi \psi \psi)_{n} \leq(\phi \psi)_{n}
$$

since every $\psi_{n}$ is an underfilter.

If, instead of (6.4) and (6.5), one makes the stronger assumption that $\phi_{n} \leq \phi_{n-1}$ and $\psi_{n} \geq \psi_{n-1}$, i.e.,

$$
\cdots \leq \phi_{3} \leq \phi_{2} \leq \phi_{1} \leq \psi_{1} \leq \psi_{2} \leq \psi_{3} \leq \cdots,
$$

then the sequences $(\phi \psi)_{n}$ and $(\phi \psi \phi)_{n}$ satisfy, besides the results stated in the previous proposition,

$$
(\xi)_{m}(\xi)_{n} \geq(\xi)_{n}, \quad m \leq n,
$$

and the sequences $(\psi \phi)_{n}$ and $(\psi \phi \psi)_{n}$ satisfy

$$
(\xi)_{m}(\xi)_{n} \leq(\xi)_{n}, \quad m \leq n
$$

To prove, e.g., the first inequality for $(\phi \psi)_{n}$ we note that

$$
(\phi \psi)_{m} \geq(\phi \phi)_{m} \geq(\phi)_{m} \geq \phi_{m} \geq \phi_{n}, \quad m \leq n \text {. }
$$

This implies that

$$
(\phi \psi)_{m}(\phi \psi)_{n} \geq \phi_{n}(\phi \psi)_{n}=\phi_{n}^{2} \psi_{n}(\phi \psi)_{n-1} \geq \phi_{n} \psi_{n}(\phi \psi)_{n-1}=(\phi \psi)_{n}
$$

6.8. Example. Assume that $\phi$ is an overfilter, $\psi$ an underfilter, and $\phi \leq \psi$. Let $N \geq 1$ be fixed, and define

$$
\phi_{n}=\phi^{N+1-n}, \quad \psi_{n}=\psi^{N+1-n}, \quad n=1,2, \ldots, N .
$$

Then $\phi_{n}, \psi_{n}$ satisfy the inequalities in (6.7). 
6.9. Example. Assume that $\alpha_{n}, \beta_{n}$ are openings and closings, respectively, and that $\xi$ is an increasing operator such that

$$
\cdots \leq \alpha_{2} \leq \alpha_{1} \leq \xi \leq \beta_{1} \leq \beta_{2} \leq \cdots
$$

If $\phi_{n}=\alpha_{n} \xi$ or $\xi \alpha_{n}$ and if $\psi_{n}=\beta_{n} \xi$ or $\xi \beta_{n}$, then $\phi_{n}, \psi_{n}$ are overfilters and underfilters, respectively (see Proposition 4.4), and (6.7) holds.

In Figure 2 we depict one particular example. Here $\xi$ is the median operator on $\mathcal{P}\left(\mathbb{Z}^{2}\right)$ using the rhombus as structuring element (origin and four horizontal and vertical neighbours), and $\alpha_{n}, \beta_{n}$ are the opening and closing, respectively, with the $(2 n+1) \times(2 n+1)$ square. Furthermore $\phi_{n}=\alpha_{n} \xi$ and $\psi_{n}=\beta_{n} \xi$. It follows easily that the conditions above are satisfied. Thus we can compute the corresponding AS-filters. The images in Figure 2 show clearly that the performance of the generalised AS-filter $(\phi \psi)_{n}$ is considerably better than that of the classical AS-filter $(\alpha \beta)_{n}$. Similarly, the performance of $(\psi \phi)_{n}$ is much better than that of $(\beta \alpha)_{n}$.

6.10. Example. Assume that $\mathcal{L}=\mathcal{P}\left(\mathbb{E}^{d}\right)$, let $(\varepsilon, \delta)$ be an adjunction, $\phi$ an overfilter, $\psi$ an underfilter, and $\phi \leq \psi$. Assume also that all these operators are translation invariant. Define

$$
\phi_{n}=\delta^{n} \phi \varepsilon^{n} \text { and } \psi_{n}=\varepsilon^{n} \psi \delta^{n} .
$$

Then $\phi_{n}=\delta \phi_{n-1} \varepsilon \leq \phi_{n-1}$, and dually, $\psi_{n} \geq \psi_{n-1}$; see Lemma 2.7. Thus (6.7) holds.

6.11. Remark. Let $\mathcal{L}$ be an arbitrary complete lattice, assume that $\phi$ is an overfilter on $\mathcal{L}$, that $(\varepsilon, \delta)$ is an adjunction on $\mathcal{L}$, and that $\phi \geq \delta \varepsilon$. Define

$$
\phi_{n}=\delta^{n} \phi \varepsilon^{n} \text {. }
$$

Then

$$
\begin{aligned}
\phi_{n} \phi_{n-1} & =\delta^{n} \phi \varepsilon^{n} \delta^{n-1} \phi \varepsilon^{n-1} \\
& \geq \delta^{n} \phi \varepsilon^{n} \delta^{n-1} \delta \varepsilon \varepsilon^{n-1} \\
& =\delta^{n} \phi \varepsilon^{n}=\phi_{n},
\end{aligned}
$$

that is, (6.4) is satisfied. Dually, if $\psi$ is an underfilter with $\psi \leq \varepsilon \delta$ and

$$
\psi_{n}=\varepsilon^{n} \psi \delta^{n},
$$

then $\psi_{n} \psi_{n-1} \leq \psi_{n}$, that is (6.5) holds.

\section{Adjunctions and filters}

If $(\varepsilon, \delta)$ is an adjunction on $\mathcal{L}$, then $\delta^{k} \varepsilon^{k}$ is an opening, and $\varepsilon^{k} \delta^{k}$ a closing, for every $k \geq 1$. The composition $\varepsilon \delta^{2} \varepsilon$ is a filter, too. For, $\left(\varepsilon \delta^{2} \varepsilon\right)^{2}=\varepsilon \delta^{2} \varepsilon^{2} \delta^{2} \varepsilon=\varepsilon \delta^{2} \varepsilon$. Here we have used that $\delta^{2} \varepsilon^{2} \delta^{2}=\delta^{2}$ since $\left(\varepsilon^{2}, \delta^{2}\right)$ is an adjunction. Note also that

$$
\delta \varepsilon \leq \varepsilon \delta^{2} \varepsilon \leq \varepsilon \delta
$$

Consider an arbitrary finite composition of $\varepsilon$ 's and $\delta$ 's. If the right-most term in such a composition is a (nonzero) power of $\delta$, then we call this composed operator of type $D$; otherwise it is said to be of type E. For example, $\varepsilon \delta^{2} \varepsilon$ is of type E. Thus an operator of type D is of the form

$$
\psi=\varepsilon^{e_{n}} \delta^{d_{n}} \cdots \varepsilon^{e_{2}} \delta^{d_{2}} \varepsilon^{e_{1}} \delta^{d_{1}} .
$$

Here the power $e_{n}$ may be equal to 0 , but all other powers are integers $\geq 1$. Dually, an operator of type E can be written as

$$
\psi=\delta^{d_{n}} \varepsilon^{e_{n}} \cdots \delta^{d_{2}} \varepsilon^{e_{2}} \delta^{d_{1}} \varepsilon^{e_{1}} .
$$

Here $d_{n}$ may equal 0 , but all other powers are $\geq 1$.

We state the main theorem of this section. Its proof will be given later in this section 

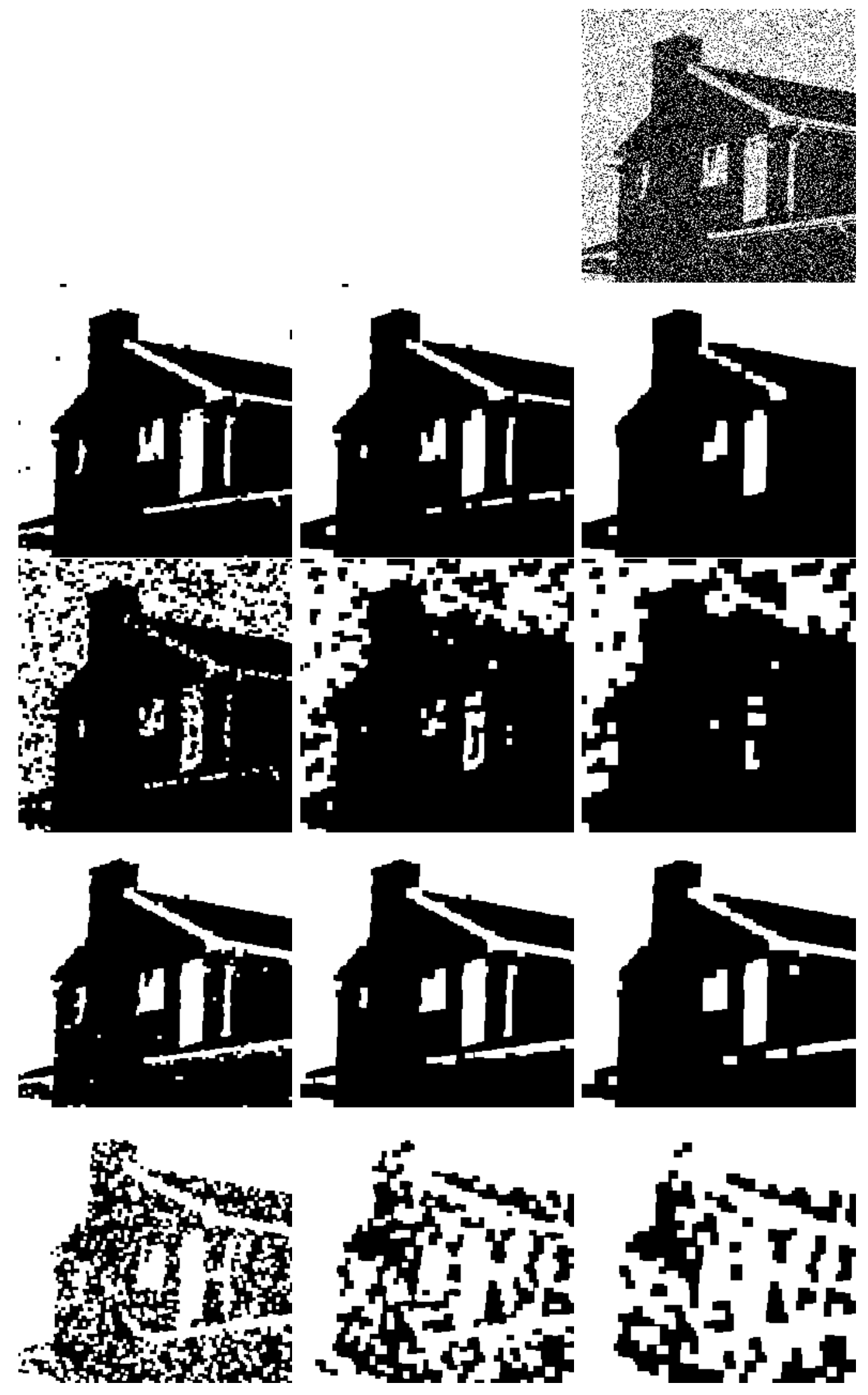

Fig. 2. Top: the original image $X$; second row: $(\phi \psi)_{n}(X)$ for $n=1,2,3$; third row: $(\alpha \beta)_{n}(X)$ for $n=1,2,3$; fourth row: $(\psi \phi)_{n}(X)$ for $n=1,2,3$; fifth row: $(\beta \alpha)_{n}(X)$ for $n=1,2,3$. 
7.1. Proposition. Assume that $(\varepsilon, \delta)$ is an adjunction on $\mathcal{L}$. Let $\psi$ be a composition of $\varepsilon$ 's and $\delta$ 's of the form (7.1) or (7.2). If

$$
\sum_{i=1}^{n} e_{i}=\sum_{i=1}^{n} d_{i}
$$

then $\psi$ is a filter.

A filter which is composed of an equal number of dilations and erosions from an adjunction is called an adjunctional filter.

The operator $\varepsilon \delta^{2} \varepsilon$ considered above is a filter, but it is neither an opening nor a closing; in fact, it is a composition of the closing $\varepsilon \delta$ and the opening $\delta \varepsilon$. In many cases, expressions of the form (7.1) (or dually (7.2)) can be simplified. For example, an expression like $\delta^{4} \varepsilon^{3} \delta^{3}$ may be replaced by $\delta^{4}$. For, $\left(\varepsilon^{3}, \delta^{3}\right)$ is an adjunction, and therefore (see Proposition 2.3(d)) $\delta^{3} \varepsilon^{3} \delta^{3}=\delta^{3}$, yielding that $\delta^{4} \varepsilon^{3} \delta^{3}=\delta \delta^{3} \varepsilon^{3} \delta^{3}=\delta \delta^{3}=\delta^{4}$. More generally, if a composition contains an expression $\delta^{d^{\prime}} \varepsilon^{e} \delta^{d}$ with $e \leq d$ and $e \leq d^{\prime}$, this can be simplified to $\delta^{d+d^{\prime}-e}$. Dually, $\varepsilon^{e^{\prime}} \delta^{d} \varepsilon^{e}$ with $d \leq e$ and $d \leq e^{\prime}$ can be simplified to $\varepsilon^{e+e^{\prime}-d}$. A composition which does not allow any further simplification is called irreducible. For example $\varepsilon^{3} \delta^{4} \varepsilon^{3} \delta^{2}$ is irreducible.

7.2. Lemma. Let $\psi$ be an irreducible composition of either type for which $\sum_{i=1}^{n} e_{i}=\sum_{i=1}^{n} d_{i}$ and $n>1$. (The latter means that the composition contains at least three terms.)

(a) If $\psi$ is of type $D($ see $(7.1))$ then $e_{1}>d_{1}$.

$\left(\mathrm{a}^{\prime}\right)$ If $\psi$ is of type $E$ (see (7.2)) then $d_{1}>e_{1}$.

Proof. We prove (a). Assume $d_{1} \geq e_{1}$. As no simplification is possible we conclude that

$$
e_{n}<d_{n}<\cdots<e_{2}<d_{2}<e_{1} \leq d_{1} .
$$

But this yields that $\sum_{i=1}^{n} e_{i}<\sum_{i=1}^{n} d_{i}$, a contradiction.

Define

$$
\alpha_{n}=\delta^{n} \varepsilon^{n} \text { and } \beta_{n}=\varepsilon^{n} \delta^{n} .
$$

\section{Proof of Proposition 7.1}

We show that every composition of type $\mathrm{D}$ can be written in the form

$$
\psi=\alpha_{a_{n}} \beta_{b_{n}} \cdots \alpha_{a_{1}} \beta_{b_{1}},
$$

where $a_{i}, b_{i}$ are integers $\geq 1$. Then Corollary 6.5 yields that $\psi$ is a filter. This would prove the proposition.

Suppose we have a type D operator given by (7.1), i.e.,

$$
\psi=\varepsilon^{e_{n}} \delta^{d_{n}} \cdots \varepsilon^{e_{2}} \delta^{d_{2}} \varepsilon^{e_{1}} \delta^{d_{1}},
$$

which is irreducible and satisfies $\sum_{i=1}^{n} e_{i}=\sum_{i=1}^{n} d_{i}$. If $n=1$, then $\psi=\beta_{d_{1}}$ and the assertion follows trivially. Now assume that $n>1$. From Lemma 7.2(a) we get that $e_{1}>d_{1}$, and thus we can write $\psi=\psi^{\prime} \beta_{d_{1}}$, where

$$
\psi^{\prime}=\varepsilon^{e_{n}} \delta^{d_{n}} \cdots \varepsilon^{e_{2}} \delta^{d_{2}} \varepsilon^{e_{1}-d_{1}} .
$$

Observe that $\psi^{\prime}$ is an operator of type $\mathrm{E}$ which contains one term less than $\psi$. Now there are two possibilities: (i) $\psi^{\prime}$ contains two terms, in which case it is an opening; (ii) $\psi^{\prime}$ contains more than two terms. In the latter case it satisfies the conditions in Lemma $7.2\left(\mathrm{a}^{\prime}\right)$, yielding that $d_{2}>e_{1}-d_{1}$. In this case we can write $\psi^{\prime}=\psi^{\prime \prime} \alpha_{e_{1}-d_{1}}$. We can repeat this procedure until we arrive at an operator consisting of two terms: this operator is an opening $\alpha_{k}$ or a closing $\beta_{k}$. 
Thus every composition as described in Proposition 7.1 can be written as a composition of $\alpha_{k}$ 's and $\beta_{k}$ 's. For example, $\varepsilon \delta^{2} \varepsilon^{3} \delta^{3} \varepsilon=\beta_{1} \alpha_{1} \beta_{2} \alpha_{1}$.

Suppose that $\psi$ is an operator of type $\mathrm{D}$ given by (7.1), then we define

$$
\tilde{\psi}=\delta^{d_{1}} \varepsilon^{e_{n}} \delta^{d_{n}} \cdots \varepsilon^{e_{2}} \delta^{d_{2}} \varepsilon^{e_{1}} .
$$

Dually, if $\psi$ is an operator of type E given by (7.2), then we define

$$
\tilde{\psi}=\varepsilon^{e_{1}} \delta^{d_{n}} \varepsilon^{e_{n}} \cdots \delta^{d_{2}} \varepsilon^{e_{2}} \delta^{d_{1}} .
$$

We show the following result.

7.3. Proposition. Let $\psi$ be an adjunctional filter.

(a) If $\psi$ is of type $D$, and if $\tilde{\psi}$ given by (7.4) is an opening, then $\psi$ is a sup-filter.

$\left(\mathrm{a}^{\prime}\right)$ If $\psi$ is of type $E$, and if $\tilde{\psi}$ given by (7.5) is a closing, then $\psi$ is an inf-filter.

Proof. Let $\psi$ be given by (7.1), then

$$
\begin{aligned}
\psi(\mathrm{id} \vee \psi) & =\varepsilon^{e_{n}} \delta^{d_{n}} \cdots \varepsilon^{e_{2}} \delta^{d_{2}} \varepsilon^{e_{1}} \delta^{d_{1}}\left(\mathrm{id} \vee \varepsilon^{e_{n}} \delta^{d_{n}} \cdots \varepsilon^{e_{2}} \delta^{d_{2}} \varepsilon^{e_{1}} \delta^{d_{1}}\right) \\
& =\varepsilon^{e_{n}} \delta^{d_{n}} \cdots \varepsilon^{e_{2}} \delta^{d_{2}} \varepsilon^{e_{1}}\left(\delta^{d_{1}} \vee \delta^{d_{1}} \varepsilon^{e_{n}} \delta^{d_{n}} \cdots \varepsilon^{e_{2}} \delta^{d_{2}} \varepsilon^{e_{1}} \delta^{d_{1}}\right) \\
& =\varepsilon^{e_{n}} \delta^{d_{n}} \cdots \varepsilon^{e_{2}} \delta^{d_{2}} \varepsilon^{e_{1}}\left(\mathrm{id} \vee \delta^{d_{1}} \varepsilon^{e_{n}} \delta^{d_{n}} \cdots \varepsilon^{e_{2}} \delta^{d_{2}} \varepsilon^{e_{1}}\right) \delta^{d_{1}} \\
& =\varepsilon^{e_{n}} \delta^{d_{n}} \cdots \varepsilon^{e_{2}} \delta^{d_{2}} \varepsilon^{e_{1}}(\mathrm{id} \vee \tilde{\psi}) \delta^{d_{1}} \\
& =\varepsilon^{e_{n}} \delta^{d_{n}} \cdots \varepsilon^{e_{2}} \delta^{d_{2}} \varepsilon^{e_{1}} \delta^{d_{1}} \\
& =\psi .
\end{aligned}
$$

Thus $\psi$ is a sup-filter. Here we have used that id $\vee \tilde{\psi}=\mathrm{id}$, since $\tilde{\psi}$ is an opening.

To check whether a composition of $\varepsilon$ 's and $\delta$ 's is an opening or a closing is easy: one has to simplify the expression until it becomes irreducible. It is a closing if the resulting irreducible expression is of the form $\varepsilon^{m} \delta^{m}$, for some $m \geq 1$, and dually, it is an opening if the resulting irreducible expression is of the form $\delta^{m} \varepsilon^{m}$.

For example, the operator $\psi=\varepsilon \delta^{2} \varepsilon$ is of type $\mathrm{E}$, and $\tilde{\psi}=\varepsilon^{2} \delta^{2}$ is a closing. Now (a') yields that $\psi$ is an inf-filter.

\section{Concluding remarks}

In this paper we have described a number of ways to construct morphological filters (or filterderivates) by composition. Among others, we have extended the class of AS-filters by taking compositions of overfilters and underfilters instead of openings and closings. The examples depicted in Figure 1 and Figure 2 show that such a generalisation may lead to considerable improvements of known filters.

A major drawback of our construction method is that it does, in general, yield filters which are not self-dual. Recall that an operator on a complete lattice with a negation $\nu: X \mapsto X^{*}$ (a negation is a decreasing bijective mapping $\nu$ with $\nu^{2}=$ id) is self-dual if $\psi^{*}=\psi$. Here $\psi^{*}(X)=\left(\psi\left(X^{*}\right)\right)^{*}$ is called the negative operator of $\psi$. For a comprehensive discussion on self-dual operators and filters the reader may refer to [3].

Consider a filter $\psi$ that is not self-dual. Let $\psi^{*}$ be the negative of $\psi$. Then $\psi \wedge \psi^{*}$ is an overfilter and $\psi \vee \psi^{*}$ is an underfilter. The so-called centre of these two operators, given by

$$
\gamma=\left(\text { id } \wedge\left(\psi \vee \psi^{*}\right)\right) \wedge\left(\psi \wedge \psi^{*}\right)
$$

is self-dual (cf. the last paragraph of Section 3). It is a filter if $\psi$ is a strong filter. For, in this case $\psi^{*}$ is strong, too. Then $\psi \wedge \psi^{*}$ is a sup-underfilter and $\psi \vee \psi^{*}$ is an inf-overfilter. Then, by Proposition 3.8, the operator $\gamma$ is a filter, presumed that the complete lattice $\mathcal{L}$ is modular. Furthermore, $\gamma$ is self-dual. Unfortunately, this result cannot be extended to arbitrary filters $\psi$, and as such it is only applicable in a very limited number of cases. 


\section{References.}

[1] Birkhoff, G. Lattice Theory, 3rd ed., vol. 25 of American Mathematical Society Colloquium Publications. American Mathematical Society, Providence, RI, 1967.

[2] Heijmans, H. J. A. M. Morphological Image Operators. Academic Press, Boston, 1994.

[3] Heijmans, H. J. A. M. Self-dual morphological operators and filters. Journal of Mathematical Imaging and Vision (to appear). CWI Research report BS-R9409.

[4] Heijmans, H. J. A. M., And Ronse, C. The algebraic basis of mathematical morphologypart I: Dilations and erosions. Computer Vision, Graphics and Image Processing 50 (1990), $245-295$.

[5] Matheron, G. Random Sets and Integral Geometry. John Wiley and Sons, New York, 1975 .

[6] Ronse, C. Why mathematical morphology needs complete lattices. Signal Processing 21 (1990), 129-154.

[7] Ronse, C., And Heijmans, H. J. A. M. The algebraic basis of mathematical morphology - part II: Openings and closings. Computer Vision, Graphics and Image Processing: Image Understanding 54 (1991), 74-97.

[8] Schonfeld, D., And Goutsias, J. Optimal morphological pattern restoration from noisy binary images. IEEE Transactions on Pattern Analysis and Machine Intelligence 13 (1991), $14-29$.

[9] Serra, J., Ed. Image Analysis and Mathematical Morphology. II: Theoretical Advances. Academic Press, London, 1988.

[10] Serra, J., And Vincent, L. An overview of morphological filtering. Circuits, Systems and Signal Processing 11 (1992), 47-108. 